\title{
Einsatzmöglichkeiten der Rückwärtssimulation zur Produktionsplanung in der Halbleiterfertigung
}

\author{
Christoph Laroque $^{1 *}$, Christoph Löffler ${ }^{1}$, Wolfgang Scholl², Germar Schneider ${ }^{2}$ \\ 1 Institut für Management und Information, Westsächsische Hochschule Zwickau, Kornmarkt 1, \\ 08012 Zwickau, Deutschland; *christoph.laroque@fh-zwickau.de \\ 2Infineon Technologies Dresden GmbH \& Co. KG, Königsbrücker Straße 180, 01099 Dresden, Germany
}

\begin{abstract}
Manufacturing is in general characterized by a growing number of customer-specific products that have to be manufactured and delivered in given lead times, ac $\neg$ cording to concrete delivery dates. Thus, highly relevant questions like "When to start a production order at latest, in order to stay within my lead time?" are answered by more or less primitive, backward-oriented plan-ning ap $\neg$ proaches and without taking into consideration uncerᄀtainty or alterna-tives. It gets more complex, if different products are to be produced and the more complex the underlying manufacturing system is (e.g. semiconductor with re-entry cycles). These questions could be answered more specifically, more detailed and more robust, if disᄀcrete, event-based simulation (DES) would be applied in a backward-oriented manner. Reseach results show, that the backward-oriented simulation approach can be in principle applied suc-cessfully for the scheduling of cusatomer-specific orders.
\end{abstract}

\section{Motivation}

Mit der fortschreitenden digitalen Transformation und der stegigen Entwicklung hin zu der Vision von Industrie 4.0 und dem Konzept einer „Smarten Fabrik“ ändern sich auch die Anforderungen an die Informationssysteme für die Arbeitsvorbereitung und operative Produktionsplanung. War hier in der Vergangenheit oft die wirtschaftlich optimale Auslastung der Produktionsketten zur Senkung der Kosten wesentliches Ziel aller Optimierung, so hat sich dies in den letzten Jahren zunehmend hin zu einer stärker kundenorientierten Fertigung verschoben, bei dem das Hauptaugenmerk auf der Einhaltung zugesagter Liefertermine durch das jeweilige Produktionsunternehmen ist. Diese müssen natürlich dennoch wirtschaftlich produziert und in möglichst kurzen Durchlaufzeiten ausgeliefert werden. Insbesondere die Einführung neuer Produkte ist bei stetig verkürzten Produktlebenszyklen eine sehr grosse Herausforderung.

Die Produktionsprozesse der im hier beschrieben Vorhaben adressierten Halbleiterfertigung gelten auch im Vergleich zu anderen Branchen als wesentlich komplexer, weil die Technologien im Mikro-und Nanometerbereich sehr sensitiv im Bezug auf die Prozessstabilität sind und sehr viele Produktionsschritte für die einzelnen Produktionslose benötigt werden (bis zu 1000 Produktionsschritte und teilweise bereits mehr). Des Weiteren müssen viele Produkte innerhalb des Produktmixes mehrfach mit hohem Automationsgrad und unter Reinraumbedingungen über spezielle Anlagen und Transportrouten prozessiert werden (Re-Entry-Cycles). Die gesamte Produktion findet dabei bereits teilweise über verschiedene Standorte hinweg statt, sodass an den vielen unterschiedlichen Prozessschritten Ausschuss von angefertigten Produkten in relevanter Größenordnung entstehen kann, der kurzfristig durch zusätzliche Einschleusungen neuer Produktionslose ausgeglichen werden muss. In der Produktionsfeinplanung ergeben sich aus dieser Kombination vielfältige Fragestellungen, die mit den existierenden Werkzeugen zur Generierung von Ablaufplänen der-zeit nicht oder nicht hinreichend gelöst werden können.

Im Rahmen des EU-ECSEL-Projektes iDEV40 ${ }^{1}$ wird die Erarbeitung von Einsatzmöglichkeiten der innovativen Methode der Rückwärtssimulation zur Planung und Steuerung von Entwicklungs- und Fertigungslosen auf Basis der diskret, ereignisgesteuerten Materialflusssimulation erarbeitet. Die prinzipielle Machbarkeit der Methode auf Fabrikebene (vgl. [1], [2]) muss hinsichtlich

\footnotetext{
${ }^{1}$ Vgl. Darstellung des Gesamtprojektes unter www.idev40.eu
} 
der Spezifika der Branche übertragen und angepasst werden. Der Beitrag beschreibt Teile der bisher erzielten Ergebnisse und damit Anwendungsmöglichkeiten der Methode sowie die nächsten Schritte zur Realisierung einer praxisnahen Anwendung zur operativen Entscheidungsunterstützung. Nach einer kurzen Darstellung des prinzipiellen Lösungsansatzes wird beispielhaft eines der erzeugten Testmodelle detaillierter beschrieben und die Ergebnisse der entsprechenden Simulationsergebnisse dargestellt. Eine Zusammenfassung beschreibt abschließend die nächsten Schritte im Projekt.

\section{Lösungsansatz Rückwärtssimula- tion}

Heutige Ziele der Produktionsplanung und -steuerung (PPS) sind wettbewerbsfähige Kosten, kurze Durchlaufzeiten, hohe Termintreue und die Erfüllung der Qualitätsanforderungen bei möglichst niedrigen Beständen. Flexilibität als Optimierungsfaktor erlebt ebenfalls eine steigende Bedeutung, wobei die jeweiligen Gewichtungen von Einsatzweck zu Einsatzzweck varriieren. Die Maximierung der Kapazitätsauslastung hat ihre große Bedeutung im heutigen Käufermarkt eingebüßt [6]. Zur Lösung dieser Planungsaufgaben kommen herkömmlich Methoden der gemischt-ganzzahlige Optimierung, unterschiedliche Heuristiken wie bspw. Tabu Search oder Simulated Annealing oder einfache Voraus- oder Rückwärtsplanung (mit oder ohne Kapazitätsbeschränkungen) zur Erzeugung erster gültiger Lösungen zum Einsatz. Abhängig vom konkreten Planungsziel wird bei diesen Verfahren (abgesehen von der Optimierung) der Planungszeitraum zeitlich vorwärts oder rückwärts betrachtet. Für die Maximierung des Durchsatzes wird der Planungszeitraum vorwärts betrachtet und es wird versucht, beginnend bei einem Startzeitpunkt, die gegebenen Aufträge möglichst schnell fertigzustellen. Bei der Terminplanung sollen bestimmte Liefertermine eingehalten werden, deshalb wird der Planungszeitraum hier häufig rückwärts geplant. Jeder Auftrag wird beginnend bei seinem Liefertermin auf der letzten Ressource eingeplant und dann wird bestimmt, wann die vorgelagerten Ressourcen von diesem Auftrag belegt werden (vgl. bspw. [3]). Ziel dieser Untersuchung ist es, für jeden Auftrag den Zeitpunkt zu erfassen, an dem ein Auftrag spätestens eingesteuert werden muss, um noch zum vereinbarten Liefertermin fertiggestellt zu werden.
Sehr viel komplexere Modelle können mit der diskreten ereignisorientierten Simulation (DES) bearbeitet werden. DES wird meist auf strategischer oder taktischer Ebene eingesetzt, beispielsweise im Rahmen der Fabrikoder Logistikplanung. Im operativen Produtionsbereich ist der Einsatz von DES zur Absicherung von konkreten Produktionsplänen oder Anlagen(um)planungen bekannt. Voraussetzung ist hier ein konkretes Produktionsprogramm, das als Eingabe für ein spezifisches Simulationsmodell dient. Ergebnis solcher Simulationen ist eine Aussage, ob das Produktionsprogramm realisierbar ist und ob alle vorher festgelegten Liefertermine eingehalten werden. Ist dies nicht der Fall, muss durch systematische Suche und Auswahl von Planvariationen (ggf. in anderen Werkzeugen oder mit anderen Algorithmen) ein durchführbarer Produktionsplan gefunden werden, dessen Validierung durch die Simulation positiv ausfällt. Modelle zur DES können aber eine sehr genaue Abbildung der Realität darstellen, sind gut zu parametrisieren und berücksichtigen die Variabilität der Realität, indem zufällige Einflüsse der Realität über stochastische Bestandteile der Modelle integriert werden können[7]. Zusätzlich können verschachtelte Ressourcenbeziehungen, Wartungsvorgänge und spezifische Ablauf-, Prioritäts-, Batch- oder Rüstregeln modelliert werden. Aus diesen Gründen sind diese Systeme eher für die Planung in der Halbleiterfertigung geeignet und werden im Rahmen dieses Vorhabens detaillierter betrachtet.

Im Allgemeinen wird die Simulation aber sowohl im Einzeleinsatz, als auch in der Kombination mit Heuristiken im Rahmen der simulationsgestützten Optimierung nur zur Untersuchung von zeitlich vorwärts gerichteten Planungsproblemen benutzt. Der Einsatz in zeitlich rückwärtsgerichteten Planungsproblemen (im Folgenden: Rückwärtssimulation) ist selten und hat sich bisher nicht in der wissenschaftlichen und industriellen Anwendung etablieren können, obwohl die Vorteile der Simulation auch in der Anwendung der rückwärtsorientierten Planung zum Tragen kommen [4][9]. Erste Anwendungsstudien, bei denen Aufträge mit Hilfe einer Rückwärtssimulation zeitlich rückwärtsgerichtet eingeplant werden, sind bereits seit mehr als 15 Jahren verfügbar. Watson et al. [11], [12], Ying und Clark [13] und Jain und Chan [5] nutzen solche Verfahren, um die Freigabezeitpunkte von Aufträgen oder Losen auch unter stochastischen Charakteristika der Modelle berechnen zu können. In Rückwärtsmodellen treten Aufträge an den Stellen in das System ein, an denen sie dieses im Vorwärtsmodell verlassen 
(Umkehrung der Quelle-Senke-Beziehung). Sie verlassen das Rückwärtsmodell an den Eintrittsstellen des Vorwärtsmodells („From product to raw material“). Die mit der Rückwärtssimulation gesuchten Freigabezeiten sind dann die Zeitpunkte, die sich ergeben, wenn die Durchlaufzeiten mit den Eintrittszeitpunkten in Beziehung gesetzt werden. Dabei ist die Rückwärtssimulation keine reine „Umkehrfunktion“ der Vorwärtssimulation. Vorwärts- und Rückwärtssimulation müssen zur selben berechneten Simulationszeit nicht denselben Zustand aufweisen [13]. Speziell die in den Modellen verwendeten Steuerungsregeln lassen sich nicht 1:1 in das entsprechende Rückwärts-Pendant übertragen. Analog zur Rückwärtsterminierung in PPS-Systemen wird auch die Rückwärtssimulation in Kombination mit vorwärts gerichteten Simulationsläufen durchgeführt, um die resultierenden Pläne nochmals abzusichern.

In der Domäne der Halbleiterfertigung konnten in den vergangenen Jahren erste Beispiele durch die Autoren erfolgreich realisiert und publiziert werden (vgl. [14]). Die dabei untersuchten Modelle hatten aber einen relativ einfachen Charakter und orientierten sich im wesentlichen an einem linearen Produktionsablauf. Spezielle Eigenschaften der Halbleiterfertigung (s.o.) wurden nicht mit abgebildet.

\section{Bisherige Ergebnisse}

Das Modell Lead Acid Battery Production der Anylogic Beispielbibliothek ${ }^{2}$ bildet eine kleine Produktionslinie von Bleiakkumulatoren ab und stellte die Grundlage für die ersten Untersuchungen der Rückwärtsmodellierung dar. Innerhalb dieses Modells kommen neben Förderbändern und Industriekränen auch fahrerlose Transportsysteme zum Einsatz, welche sich bei dem Transport von Elektrodenchargen und Batterien sowohl entlang von Führungslinien als auch im freien Raum bewegen können. Neben der Modellierung der Prozesskette umfasst das Modell eine zweidimensionale als auch eine dreidimensionale Darstellung. Das Modell wurde im Rahmen des Vorhabens mehrfach hinsichtlich verschiedener Charakteristika der Halbleiterfertigung erweitert, so dass neben parallelen Bearbeitungsschritten, den oben beschriebenen Re-Entry-Cycles auch Batch-Prozesse in dem Modell abgebildet werden. Das Modell wurde zur

\footnotetext{
${ }^{2}$ Das ursprüngliche Modell ist frei unter https://cloud.anylogic.com/ verfügbar
}

Erhöhung der Komplexität noch gespiegelt und besitzt in der letzten Ausbaustufe ein gemeinsames Auslieferungslager in der räumlichen Mitte der Fertigung. Das abgebildete Auftragsprogramm im Modell wurde sukzessive um verschiedenen Prioritätsstufen der Aufträge und verschiedene Losgrößen für die Aufträge ergänzt. Abbildung 1 zeigt die grafische Darstellung der finalen Ausbaustufe (oben) sowie die resultierende Materialflussstruktur des Modells (unten).

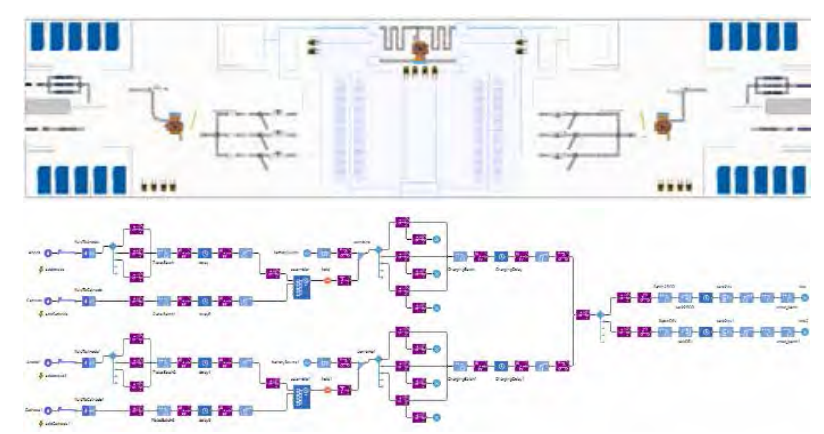

Abbildung 1: Modellstruktur des erweiterten Beispiels zur Batteriefertigung

Sowohl die Vorwärtsmodellierung als auch die Rückwärtsmodellierung wurden in experimentellen Versuchen hinsichtlich der Auswirkungen sich verändernder Parameter betrachtet (Sensitivitätsanalysen). Für das Vorwärtsmodell konnte somit in einem ersten Schritt eine vernünftige Einstellung der Parameter erreicht werden: Mit 150 Elektroden pro Los in der Produktion konnten 668 Stunden Simulationszeit insgesamt 388 Batterien für die im Modell hinterlegten Aufträge produziert werden. Bei einer Losgröße von 50 Elektroden wäre dieselbe Anzahl Batterien erst nach 672 Stunden erfüllt worden. Für die Rückwärtsmodellierung konnten weitestgehend die gleichen Parameter angesetzt werden, wobei entsprechende Parameter zur Rohstoffverfügbarkeit (,filling quantity` als Füllmenge des flüssigen Metalls, welches in der Vorwärtsmodellierung als Quelle dient) durch korrespondierende Parameter (hier ,plates batch`als Batchgröße des entsprechenden Prozesses) ersetzt wurden. Auch die entsprechenden Steuerungsregeln des Vorwärtsmodells mussten im Rahmen der Modellierung des Rückwärtssimulationsmodells entsrpechende umgekehrt werden. Abbildung 2 zeigt exemplarisch die Auswertung eines Simulationslaufs, wobei die einzelnen Ausliefe- 
rungstermine für Produktionslose (PROD) und Entwicklungslose (DEV) separat ausgewiesen werden.

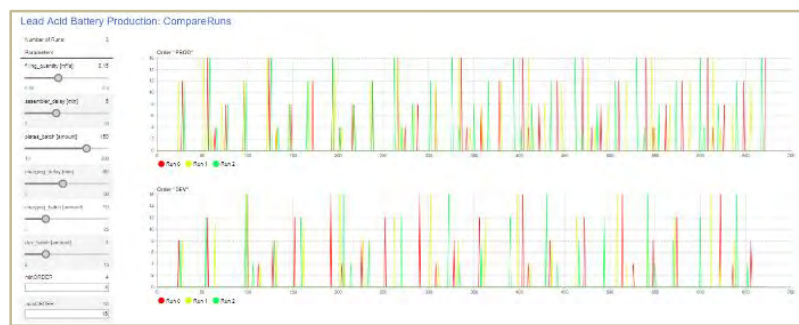

Abbildung 2:Grafische Auswertung des Vorwärtssimulationsmodells für ein spezifisches Parameter-Setting

Nach der Modellierung der beiden Einzelmodelle für Vorwärts- und Rückwärtssimulation (Abbildung 3 zeigt den resultierenden Materialfluss für das entsprechende Rückwärtsmodell) sollten die beiden Modelle in einem weiteren Schritt gegeneinander validiert werden.

Im Fall des hier dargestellten Beispiels wurde dazu auf Basis der Ergebnisse der Sensitivitätsanalyse des Vorwärtsmodells ein Auftragsprogramm selektiert, dass eine relativ hohe Auslastung der Fabrik zur Folge hat. Für dieses Auftragsprogramm wurden zunächst mittels einer ersten Vorwärtssimulation (Simulaton 0) Fertigstellungstermine erzeugt. Diese wurden als Eingabeparameter für dasselbe Auftragsprogramm in der korrespondierenden Rückwärtssimulation als Liefertermine übernommen. Als Ergebnis der Simulationsläufe der Rückwärtssimulation (R-Simulation 1) entstehen dann für dieses Auftragsprogramm Einschleustermine als Ergebnis des Planungslaufes. Für die meisten Aufträge unterscheiden sich diese Termine allerdings von ihrer ursprünglichen Einsteuerung im Vorwärtslauf 0.

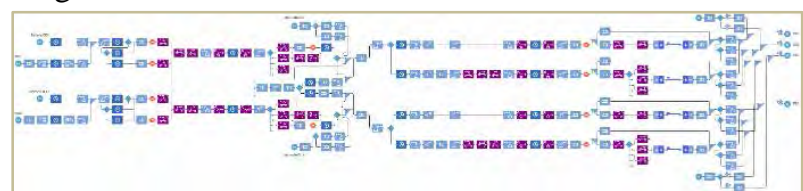

Abbildung 3:Materialflussstruktur des Rückwärtssimulationsmodells in der finalen Ausbaustufe

Als eine erste Validierung des Rückwärtsmodells wurden daher die Einschleustermine der Rückwärtssimulation erneut in das Vorwärtsmodell übernommen, simuliert und die erzielten Fertigstellungstermine (Simulation 2) mit den ursprünglichen Lieferterminen (aus Simulation 0) verglichen. Die erzielten Ergebnisse zeigen, dass die über die Rückwärtssimulation erzeugten Einschleustermine in $>95 \%$ aller Aufträge auch die pünktliche Einhaltung der Liefertermine zur Folge haben. Die beiden Modelle verhalten sich somit konsistent zueinander und konnten im Anschluss um stochastische Einflüsse in beiden Modellen ergänzt werden. Nachfolgend wurden die entsprechenden Experimente mit den stochastischen Modellen wiederholt, wobei hier natürlich auf eine entsprechende stochastische Breite der einzelnen Simulationsexperimente Rücksicht genommen wurde. Die jeweils erzielten Ergebnisse der Simulationsläufe (vgl. bspw. Abbildung 4) wurden zusammengetragen und ausgewertet. Insgesamt ist dazu festzuhalten, dass die Konstenz zwischen den beiden jeweiligen Simulationsmodellen erhalten blieb, wenn die Ergebnisse auch einen etwas schlechteren Erfüllungsgrad bei der Validierung der Einschleustermine aus der Rückwärtssimuation durch die Simulation 2 zur Folge hatten. Eine Liefertermintreue > 85\% konnte jedoch in allen Simulationsexperimenten nachgewiesen werden.

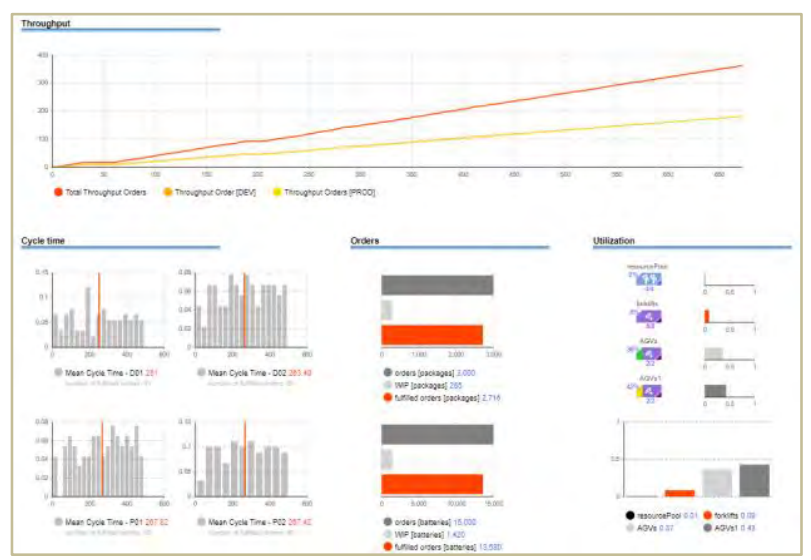

Abbildung 4: Ergebnisübersicht der Rückwärtssimulation mit stochastischen Einflüssen

Logisch gut nachzuvollziehen ist überdies, dass die Integration von Maschinenausfällen und anderen stochastischen Einflüssen wie Bearbeitungszeiten einzelner Maschienen einen merklichen Einfluss auf Gesamtdurchsatz (Total Throughput) beider Modelle aufgezeigt hat. Die erzielten Ergebnisse haben aber in einem ersten Schritt im Rahmen des Vorhabens die prinzipielle Anwendbarkeit der Methode auch mit komplexeren Materialflussstrukturen nachgewiesen und sind Basis für weitere Entwicklungen im Projekt, die im nächsten Abschnitt kurz beschrieben werden.

\section{Zusammenfassung und Ausblick}

Die mit dem erweiterten Beispiel erzeugen Ergebnisse zeigen, dass der methodische Ansatz zur Generierung eines Produktionsplans durch Rückwärtssimulation 
prinzipiell auch unter den Spezifika der Halbleiterfertigung und unter Berücksichtigung stochastischer Einflüsse funktioniert und vielversprechende Ergebnisse liefern kann. Die hier vorgenommene Validierung diente nur in einem ersten Schritt dazu, eine prinzipielle Konsistenz der beiden Modelle nachzuweisen.

Im eigentlichen Vorhaben sind weitere Schritte zur Evaluation der Methode und ihrer Einsatzgrenzen im beschriebenen Einsatzgebiet geplant. In einem ersten Schritt wird für ein weiteres, komplexeres Simulationsmodell des Projektpartners das korrespondierende Rückwärtssimulationsmodell erzeugt und die Experimente wiederholt. Im besten Fall zeigt sich hier eine Bestätigung der Ergebnisse. Das Modell entspricht einem realen Produktionsprozess und schlägt damit die Brücke von einem Testmodell mit entsprechenden Branchencharakteristika zu einem möglichen realen Einsatz der Methode für „real-world-problems“.

Hierfür ist in einem weiteren Schritt aber zunächst die Lösungsgüte der aus der Rückwärtssimulation ermittelten Einschleusplanung nicht nur hinsichtlich der prinzipiellen Erfüllbarkeit des Auftragsprogramms zu überprüfen, sondern auch mit konkurrierenden Planungsverfahren der kapazitätsbeschränkten Rückwärtsterminierung etc. Die Autoren erwarten, dass der Einsatz der Simulationsmethode einfachen Ansätzen und Heuristiken zur Planung überlegen sein sollte, weil viele der Kapazitätsbeschränkungen in dem Materialflussmodell schon berücksichtigt werden. Mathematisch optimierende Verfahren können sollten sich hinsichtlich der Lösungsgüte durchsetzen können, erzeugen ihrerseits aber einen erheblichen Aufwand zur Modellierung und Berechnung und können die stochastischen Einflüsse des realen Modells vermutlich auch nur unzureichend abbilden. Hier werden weitere Untersuchungen in der Zukunft hoffentlich genauere Vor- und Nachteile der Rückwärtssimulation benennen können.

\section{Aknowledgements}

This work has been partially funded by the European project iDEV40. The iDev40 project has received funding from the ECSEL Joint Undertaking (JU) under grant agreement No 783163. The JU receives support from the European Union's Horizon 2020 research and innovation programme. It is co-funded by the consortium members, grants from Austria, Germany, Belgium, Italy, Spain, and Romania.

\section{References}

[1] Arakawa, M.; Fuyuki, M.; Inoue, I.: A Simulation-based Production Scheduling Method for Minimizing the Duedate-deviation, International Transactions in Operational Research, Vol. 9 Issue 2, S. 153-167, 2002

[2] Graupner, T. D.; Bornhäuser, M.; Sihn, W.: Backward simulation in food industry for facility planning and daily scheduling, 16th European Simulation Symposium [ESS 2004], SCS Press, 2004

[3] Hopp, W. J.; Spearman, M. L.: Factory Physics, McGraw-Hill, New York, 2001

[4] Huang, C.; Wang, H.: Backward Simulation with Multiple Objectives Control, Proceeedings of the International MultiConference of Engineers and Computer Scientists, 2009

[5] Jain, S.; Chan, S.: Experiences with Backward Simulation Based Approach for Lot Release Planning, Winter Simulation Conference, S. 773-780, 1997

[6] Kurbel, K.: Produktionsplanung und -steuerung im Enterprise Resource Planning und Supply Chain Management, Oldenbourg Verlag, 2005

[7] Law, A.; Kelton, D.: Simulation Modeling and Analysis, McGraw Hill, 2000

[8] Rossi, H.: Ein heuristisches Dekompositionsverfahren für mehrstufige Losgrößenprobleme, Dissertation, Freie Univ. Berlin, 2003

[9] Schumacher, R.; Wenzel, S.: Der Modellbildungsprozess in der Simulation, In: Wenzel, S. (Hrsg.): Referenzmodelle für die Simulation in Produktion und Logis $\neg$ tik, S. 5-11, SCS-Europe BVBA, Gent, Belgien, 2000

[10] Tempelmeier, H.; Günther, H.-O.: Produktion und Logistik, 7. Auflage, Springer, Berlin, 2007

[11] Watson, E. F.; Medeiros, D. J.; Sadowski, R. P.: Generating Component Release Plans with Backward Simulation, Proceedings of the 1993 Winter Simulation Conference, S. 930-938, 1993

[12] Watson, E. F.; Medeiros, D. J.; Sadowski, R. P.: A simulation-based backward planning approach for order-release, Proceedings of the 29th Conference on Winter Simulation, Atlanta, Georgia, ACM Press, New York, NY, S. 765-77, 1997

[13] Ying, C. C.; Clark, G. M.: Order release planning in a job shop using a bidirectional simulation algorithm, Proceedings of the 26th Conference on Winter Simulation, Orlando, Florida, Society for Computer Simulation International, S. 1008-1012, 1994

[14] Scholl, Wolfgang; Laroque, Christoph; Weigert, Gerald: Evaluations on Scheduling in Semiconductor Manufacturing by Backward Simulation. In: Proceedings of the 2014 Winter Simulation Conference, Dec. 2014, Omnipress 